\title{
Role of Mitochondrial Therapy for Ischemic-Reperfusion Injury and Acute Kidney Injury
}

\author{
Navjot Pabla ${ }^{a} \quad$ Amandeep Bajwa ${ }^{b, c}$ \\ aDivision of Pharmaceutics and Pharmacology, College of Pharmacy \& Comprehensive Cancer Center, Ohio State \\ University, Columbus, OH, USA; ${ }^{\text {T}}$ Transplant Research Institute, James D. Eason Transplant Institute, Department \\ of Surgery, Department of Microbiology, Immunology, and Biochemistry, College of Medicine, The University of \\ Tennessee Health Science Center, Memphis, TN, USA; 'Department of Genetics, Genomics, and Informatics, College \\ of Medicine, The University of Tennessee Health Science Center, Memphis, TN, USA
}

\section{Keywords}

Acute kidney injury · Mitochondria $\cdot$ Ischemia reperfusion injury

\begin{abstract}
Acute kidney injury (AKI) is a common clinical disorder associated with decline in renal function because of ischemic and nephrotoxic insults. The pathophysiology of AKI involves multiple cellular mechanisms, such as kidney parenchymal cell (epithelial and endothelial) dysfunction and immune-cell infiltration. Mitochondrial injury which causes ATP depletion and triggers apoptosis and necrosis is at the heart of ischemia reperfusion injury (IRI). Pharmacological (SS-31 or MitoQ), cellular (dendritic cells or mesenchymal stem cells), or genetic strategies that either directly or indirectly preserve mitochondrial integrity and function have been shown to mitigate IRI-linked AKI in preclinical models. Interestingly, isolated mitochondria have been recently shown to be taken up by various mammalian cells resulting in incorporation of transplanted mitochondria into the endogenous mitochondrial network of recipient cells and contributing to protection from ischemic injury in various pre-
\end{abstract}

clinical models of ischemia including the heart, liver, and kidneys. The mini review summarizes the current available therapeutic strategies that improve kidney function by targeting mitochondria health.

(c) 2021 S. Karger AG, Basel

\section{Introduction}

Kidney ischemia-reperfusion injury (IRI) is a major cause of acute kidney injury (AKI), which in addition to kidney transplantation occurs in various other clinical settings such as cardiac surgery, sepsis, and shock. The pathophysiology of IRI is complex and involves various aspects including hypoxic injury that results in production of reactive oxygen species, triggering a cycle of cell

Contribution from the AKI and CRRT 2021 Symposium at the 26th International Conference on Advances in Critical Care Nephrology, A Virtual/Hybrid Event from San Diego, CA, USA, February 28-March 5, 2021. This symposium was supported in part by the NIDDK funded University of Alabama at Birmingham-University of California San Diego O'Brien Center for Acute Kidney Injury Research (P30DK079337). 
death and inflammation between epithelial and immune cells. In addition to their functional roles as metabolic energy producers, mitochondria can also regulate cell death. IRI involves an intricate cascade of events at the mitochondrial level that includes rapid loss of energy, decrease in mitochondrial membrane potential, and loss in ionic hemostasis and culminates in ROS production and cell death. These observations suggest that mitochondria are critical organelles that undergo major pathophysiological changes during IRI. Importantly, mitochondria have a critical role not only in initiation and progression of ischemic injury but also are involved in recovery and processes that contribute to progression to chronic kidney disease. Multiple mitochondria-targeted pharmacological (SS-31 [1] or MitoQ), cellular (mesenchymal stromal cells [2] or bone marrow-derived dendritic cells [3]), and recently mitochondria transplant [4] therapeutic strategies have been tested to improve mitochondrial health to ultimately reduce dysfunction in preclinical models of kidney IRI. Although these pharmacological and cellular therapeutics have shown some benefits in preserving kidney health, their progression to use in a clinical setting is limited due to bioavailability and offtarget adverse effects. Additionally, these studies lack a larger animal model (pigs) to further demonstrate their potential use, especially if direct delivery to kidneys is needed. Therapeutic use of mitochondrial transplantation to prevent injury has been demonstrated in various preclinical models of ischemic injury in multiple organs including the heart, liver, kidney, and lung. The dosing and treatment strategies of these studies are summarized in the current review.

\section{Involvement of Mitochondria in Kidney IRI}

Under normal healthy states, the electron transport along the electron transport chain (ETC) is coupled to oxidative phosphorylation for producing ATP. Water is the ultimate byproduct of the oxygen consumed in the ETC that generates electrons as it moves through complex I to complex IV. The small amounts of ROS produced in ETC are needed to maintain the redox state of cells and is crucial for the function of various enzymes. In ischemia, there is a decrease in activity at various complexes that results in electron leak and reduces oxygenforming superoxide radicals when oxygen is available upon reperfusion, ultimately reducing the amount of ATP available. Within the kidneys, the proximal tubules (PTs) have the highest density of mitochondria, and PT epithelial cells are heavily dependent on oxidative phosphorylation for carrying out normal fluid and solute transport. During ischemia, in addition to diminished mitochondrial function, ultrastructural analysis reveals fragmented mitochondria within 15 min of reperfusion, ultimately resulting in mitochondria swelling and disruption of tightly packed cristae $[5,6]$. These pathophysiological changes associated with IRI indicate that mitochondrial morphology and function are highly coordinated and can rapidly respond to metabolic and cellular stress. The pathogenesis of kidney IRI involves multiple complex interactions between kidney parenchymal cells and infiltrating immune cells [7]. In response to either ischemic injury or toxins, the degradation of the cellular functions can be triggered by the release of ROS from damaged mitochondria. Structural damage to mitochondria occurs within few minutes of reperfusion that leads to release of cytochrome $\mathrm{c}$ and the further release of mitochondrial DNA that can further trigger apoptosis [8]. In mouse models of kidney IRI, PT mitochondrial membrane potential decreases quickly, resulting in shorter fragmented mitochondria than in sham-operated mice. To observe these structural changes in PT mitochondria, we generated PT-specific mitochondria reporter mice to use as a tool to assess mitochondria morphology in various mouse models of AKI. Interestingly, these mice can also be used to evaluate the released mitochondria after injury in various other compartments including the spleen, liver, and urine. Thus, this further allows for evaluation of how targeted release of mitochondria from the damaged organ may influence distal organ injury due to its ability to act as mitochondria-damage-associated molecular pattern. PepcKCre mice (a gift from Dr. Volker Haase, Vanderbilt University) were bred to the Pham ${ }^{\mathrm{fl} / \mathrm{fl}}$ (purchased from Jackson Laboratory) to produce the transgenic PepcKCrePham ${ }^{\mathrm{fl} / \mathrm{fl}}$ mice that have PT mitochondria labeled with a fluorescent green tag. These mice were used for either sham or bilateral ischemia of $26 \mathrm{~min}$ with $24 \mathrm{~h}$ of reperfusion. The blood was collected under anesthesia from the retro-orbital sinus, and plasma creatinine $(\mathrm{mg} / \mathrm{dL})$ was determined by using an enzymatic method from the manufacturer's protocol (Diazyme Laboratories, Poway, CA, USA), and blood urea nitrogen (BUN) and creatinine measurements were performed using the QuantiChrom Urea Assay Kit (DIUR-100). Compared to sham mice, IRI results in a significant raise in plasma creatinine (Fig. 1a) and BUN (Fig. 1b). For histology, kidneys were fixed overnight in 10\% formalin and embedded in paraffin. Kidneys were prepared for H\&E. For quantification of tubular injury score (acute tubular 
Fig. 1. Mitochondrial damage in kidney IRI in PepckCrePham ${ }^{\mathrm{fl} / \mathrm{fl}}$ mice. Mice underwent 26 min of ischemia with $24 \mathrm{~h}$ of reperfusion. a Plasma creatinine in sham and IRI mice. $\mathbf{b}$ BUN in sham and IRI mice. c ATN in sham and IRI mice. $\mathbf{d} \mathrm{H} \& \mathrm{E}$ in sham and IRI mice, Scale bar, $100 \mu \mathrm{m}$. e Mitochondrial damage in sham and IRI mice $*^{*}=$ apoptotic/necrotic tubule), Scale bar, 20 $\mu \mathrm{m}$. f Kidney section labeled with CD13 (red), CD31 (magenta), and endogenous mitochondria (green), Scale bar, $100 \mu \mathrm{m}$. ${ }^{* * *} p<0.001$. IRI, ischemia-reperfusion injury; BUN, blood urea nitrogen; ATN, acute tubular necrosis.
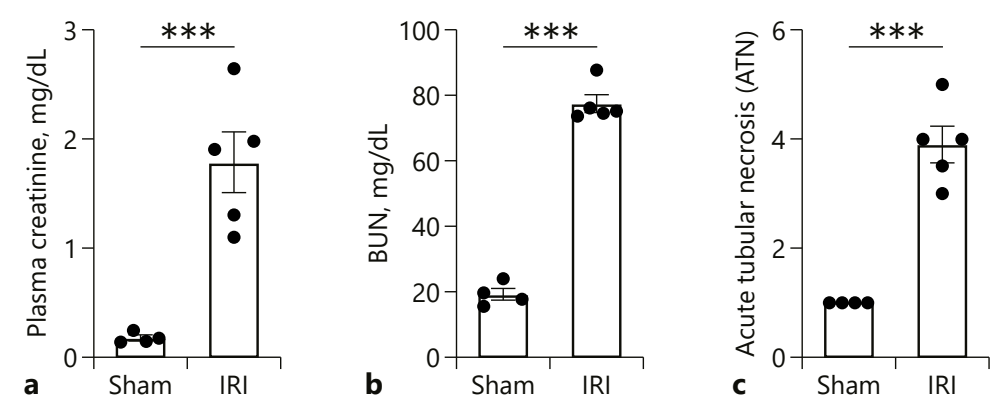

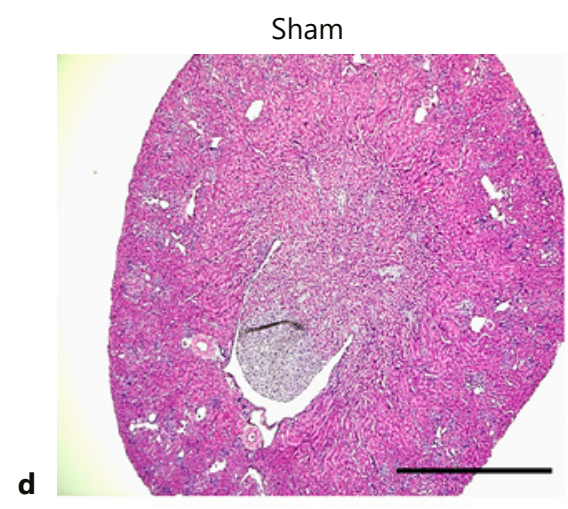

Sham

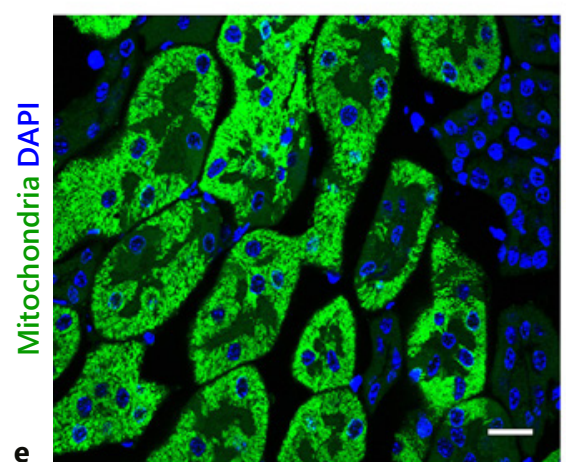

e

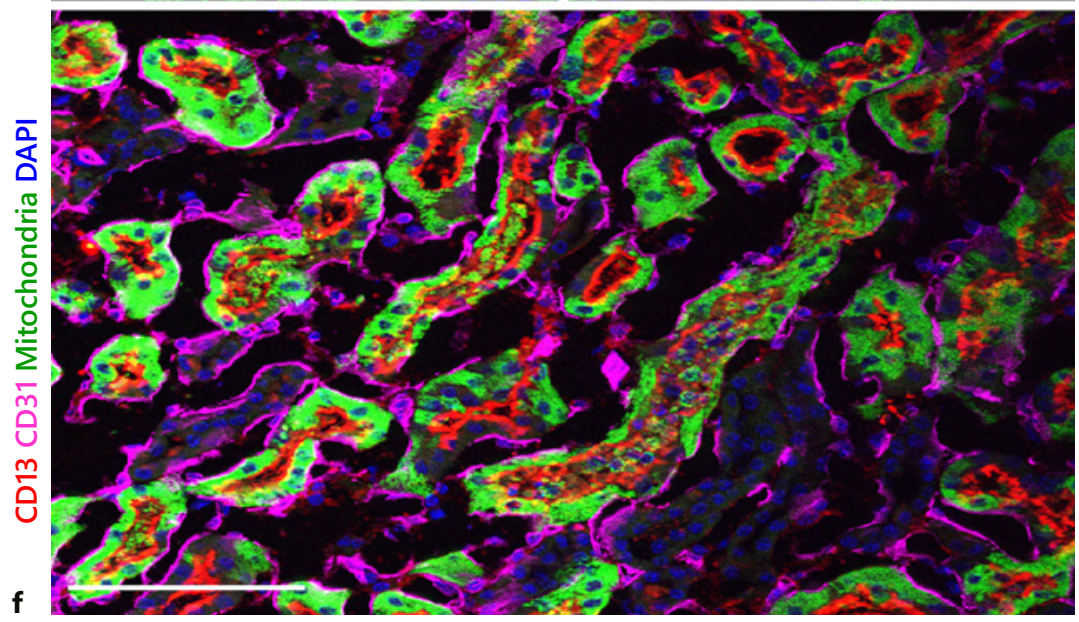

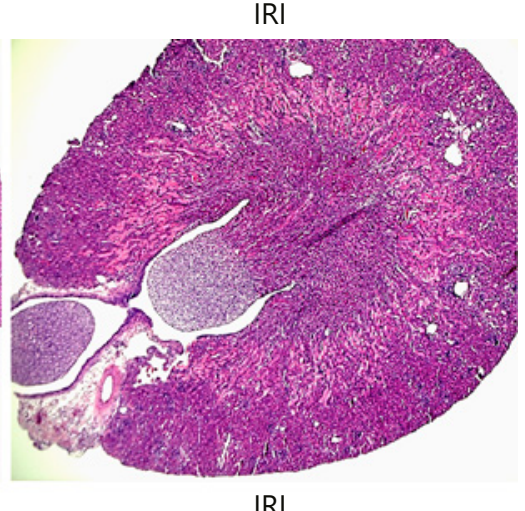

IRI

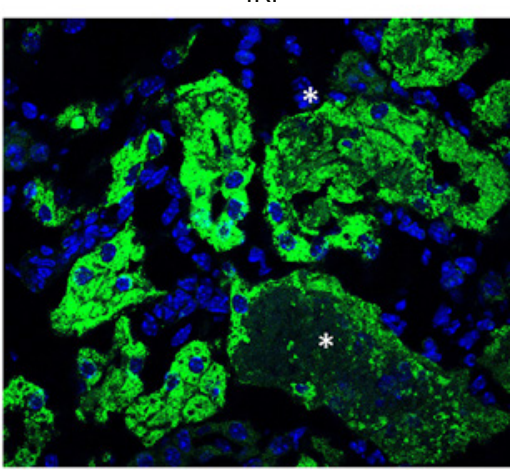


necrosis), sections were assessed by counting the percentage of tubules that displayed cell necrosis, loss of brush border, cast formation, and tubule dilation as follows: $0=$ normal; $1=<10 \% ; 2=10-25 \% ; 3=26-50 \% ; 4=51-75 \%$; and $5=>75 \%$. Five to 10 fields from each outer medulla were evaluated and scored in a blinded manner. Kidneys of IRI mice had increased acute tubular necrosis compared to sham mice (Fig. 1c) and representative $H \& E$ from sham and IRI kidney (Fig. 1d). Additionally, changes in mitochondria morphology in PepcKCrePham ${ }^{\mathrm{f} / \mathrm{fl}}$ mice shown in sham mice mitochondria are uniformly aligned in the PTs, whereas the mitochondria morphology is disrupted after IRI (Fig. 1e). The presence of the mitochondria signal (green) was further confirmed to be localized in the PT segment of the kidney in the PepcKCrePham ${ }^{\mathrm{fl} / \mathrm{fl}}$ mice by labeling with CD13 against the PT brush border and CD31 (PECAM) for the endothelium (Fig. 1f).

\section{Mitochondrial Therapy and IRI}

There are few studies to date in various preclinical models of IRI that demonstrate mitochondrial transplantation can be used as a therapeutic modality to alleviate or treat IRI-related dysfunction. A comprehensive review of the models, sources, doses, and route of delivery of isolated mitochondria in various models is listed below.

\section{Heart}

In a series of studies, the McCully's group has demonstrated that heart IRI can be significantly improved by transplant of isolated mitochondria. Overall, in the heart IRI models (swine and rabbits), their group demonstrates that injected mitochondria are specifically taken up by cardiomyocytes and contribute to enhanced oxygen consumption, high-energy phosphate synthesis, overall reduced cytokine production, preserved myocardial energetics and cell viability, and overall enhanced post-infarct cardiac function. The McCully group in 2017 reported the first clinical application of therapeutic mitochondria in pediatric patients with regions of myocardial akinesis or hypokinesis. In these 5 patients, viable mitochondria were isolated from an autologous $6 \times 6-\mathrm{mm}$ piece of healthy rectus abdominis and injected at a dose of $1 \times 10^{8}$ $\pm 1 \times 10^{5}$ with $1 \times 10^{7} \pm 1 \times 10^{4} / 0.1 \mathrm{~mL}$ per site [9]. Four of five patients with single mitochondria transplant, although at multiple sites, improved ventricular function, and these patients were successfully taken off from extracorporeal mechanical support.

\section{Source}

Mitochondria were isolated from muscle (pectoralis major) by a standard protocol developed by the McCully group that takes $30 \mathrm{~min}$ without any differential centrifugation steps for all in vivo studies [10].

\section{Model}

Rabbit heart IRI ( $30 \mathrm{~min}$ of ischemia with $2 \mathrm{~h}$ to 28 days of reperfusion). Isolated mitochondria $\left(9.7 \times 10^{6} \pm\right.$ $1.7 \times 10^{6} / \mathrm{mL}, 1.2 \times 10^{6} / 0.1 \mathrm{~mL}$ at 8 sites) were injected at the time of reperfusion [11]. Swine heart IRI (25 min of ischemia with 28 days of reperfusion). Isolated mitochondria $\left(9.9 \times 10^{7} \pm 1.4 \times 10^{7} / \mathrm{mL}, 1.3 \times 10^{7} / 0.1 \mathrm{~mL}\right.$ at 8 sites $)$ were injected at time of reperfusion [12]. All mitochondria directly injected into the heart at the regional ischemia sites.

\section{Kidney}

In kidney IRI, 2 recently published studies have demonstrated in small and large animal models the therapeutic use of isolated healthy mitochondria. Our collaborative work with McCully's group used a large swine model [4] and rats [13]. In the larger animal model, Doulamis et al. [4], concluded that injected mitochondria significantly protected kidneys from ischemic injury with improved kidney function, less histological damage, lower coagulative necrosis of the PTs, and lower kidney levels of IL6 [4]. In rats, mitochondria treated animals had significantly lower creatine and BUN starting at $12 \mathrm{~h}$ of reperfusion and remained significant until $72 \mathrm{~h}$. Interestingly, both vehicle and mitochondria treated animals had similar levels of creatinine and BUN at the 1 week time point. Overall, the mitochondria treated rats had significantly higher kidney expression of Ki67, PCNA, aquaporin 1 (AQP1, PT marker) and lower levels of Kim1, cystatine C, and TUNEL [13]. Both set of studies concluded by stating that direct injection of isolated healthy mitochondria significantly protects the kidneys from IRI and potentially helps with repair by increasing the proliferative capacity of the injury epithelium.

\section{Source}

Swine, mitochondria were isolated from sternocleidomastoid muscle [10]. Rats, mitochondria were isolated from pectoralis major muscle [10].

\section{Model}

Swine kidney IRI ( $60 \mathrm{~min}$ of ischemia with $24 \mathrm{~h}$ of reperfusion). Isolated autologous mitochondria $\left(1 \times 10^{9} / 6\right.$ $\mathrm{mL}$, single $\times 1$ or multiple $\times 3$ ) were injected intra-arterial 
at time of reperfusion [4]. Rat kidney IRI (Unilateral, right-kidney nephrectomy and 45 min of left-kidney ischemia with up to 1 week of reperfusion). Isolated mitochondria $\left(7.5 \times 10^{6} / 1 \mathrm{~mL}, 0.4 \mathrm{~mL}\right.$ injected $)$ were injected into the renal artery at the time of reperfusion [13].

\section{Liver}

In the study by Lin et al. [14], it was demonstrated that rats treated with freshly isolated mitochondria $45 \mathrm{~min}$ after liver IRI are significantly protected compared to vehicle-treated mice. Rats treated with mitochondria had less liver injury with lower ALT levels, less congestion hemorrhage, necrosis of the hepatocytes, and less TUNEL-positive cells.

\section{Source}

Mitochondria isolated from the rat liver by a standard differential centrifugation method.

\section{Model}

Rat liver IRI ( 45 min of ischemia with $4 \mathrm{~h}$ of reperfusion). Isolated mitochondria $\left(7.7 \times 10^{6} \pm 1.5 \times 10^{6} / \mathrm{mL}\right)$ were injected through an intrasplenic injection at the time of reperfusion.

\section{Lung}

In this study by Zhu et al. [15], the author used a rat hypoxic pulmonary hypertension model to assess the therapeutic use of mitochondria transplantation. Rats treated with mitochondria had reduced chronic hypoxiainduced pulmonary vascular remodeling that resulted in prevention of pulmonary hypertension. Additionally, the current study also demonstrated that mitochondria transplantation can also be used to treat rats that had established chronic hypoxia.

\section{Source}

Smooth-muscle cells from explanted pulmonary and femoral arteries. The methods used for isolating mitochondria were not listed.

\section{Model}

Rats were housed in a hypoxic chamber $\left(10 \% \mathrm{O}_{2}\right)$ for $8 \mathrm{~h}$ a day for 4 weeks. The rats were intravenously treated with $2.25 \times 10^{8}(\sim 2 \mu \mathrm{g}$ protein $)$ mitochondria every other day for 2 weeks, either 2 weeks or 4 weeks after start of study.

\section{Conclusion}

The potential use of mitochondria as a treatment modality has been demonstrated to improve disease outcomes in various preclinical models. Unlike mitochondria-centric pharmacological and cellular treatment strategies, current studies using exogenous mitochondria have reported no adverse side effects. Unanimously, one of the limitations acknowledged by all studies was that exogenous mitochondria must be freshly isolated, kept cold, and utilized within a few hours. The use of isolated viable healthy mitochondria as a therapeutic modality to supplement and functionally improve and potentially replace damaged mitochondria is a viable option. Labeled and injected mitochondria either via a local or systemic injection are taken up by various cell types. Viability of mitochondria is critical as nonviable or damaged mitochondria and mitochondrial products (DNA, RNA, protein, ATP, and complexes) do not provide any protection [16]. The injected mitochondria improve the energetics of the recipient cells [11], induces mitochondrial biogenesis (activates Pgc1a, Bajwa unpublished observations), increases proliferative capacity [13], and decreases inflammatory cytokines overall. Interestingly, some critical questions remain. How do exogenous mitochondria get into the cells? In an in vitro setting, the use to inhibitors of macropinocytosis or a macropinocytosis-like mechanism [17] or microtubules/tunneling nanotubes or gap junctions [18] partially blocks mitochondria update. How long do the injected mitochondria last? Although difficult, these studies can be done by taking advantage of the fact that mitochondria come with their own DNA, so using xenograft mitochondrial transplants to evaluate the location of systemically injected mitochondria could be utilized for addressing both the where and how long does injected mitochondria last. These studies also have additional limitations as this technique will only allow detection of injected mitochondrial DNA and cannot be used to test if injected mitochondrial is functional. Depending on the disease model, how often does the mitochondria need to be injected? In acute settings, a single dose of mitochondria seems to be adequate, but no current studies have evaluated the use of mitochondria in chronic settings. These low doses of mitochondria inhibited acute hypoxia-triggered pulmonary vasoconstriction and attenuated hypoxia-induced vascular remodeling although the signal associated with systemic mitochondria injection was found in the kidney, liver, and spleen. Does the route of injection matter? In most of the preclinical ischemic rodent models except for the heart, the isolated exogenous mitochondria are injected systemically via an intravenous 
route. However, use of larger animal models could be tested to use localized delivery to the target organ as recently demonstrated by Doulamis et al. [4] using an intra-arterial injection in a pig kidney IRI model of AKI. Localized delivery of mitochondria could potential also be beneficial as these interventions may require a lower dose of isolated mitochondria. Although recently studies have added to our current knowledge of how the mitochondria transplantation could be utilized as a potential therapeutic strategy, many other questions remain. What is the optimal dose of mitochondria that is needed for clinical use? Does the source of mitochondria matter? Does it induce a proinflammatory response, autologous versus syngeneic versus allogenic versus xenogeneic? All published studies use freshly isolated mitochondria within an hour of isolation. Can mitochondria be preserved after isolation? Having mitochondria that can be available for transplantation as an off-the-shelf treatment strategy could revolutionize the way we use it as a therapeutic modality to treat diseases. Finally, transplantation of viable healthy mitochondria provides a novel therapeutic treatment option to prevent and treat and to improve disease progression in various preclinical models. These treatment options using organelles to improve disease outcomes could have a significant clinical impact.

\section{Acknowledgment}

Research reported in this work was supported by the National Institute of Diabetes and Digestive and Kidney Diseases of the National Institutes of Health (NIH) under award number R01DK117183 (A.B.). The content is solely the responsibility of the authors and does not necessarily represent the official views of the National Institutes of Health.

\section{Conflict of Interest Statement}

The authors declare no conflict of interest.

\section{Funding Sources}

This study received financial support through NIH award R01DK117183 (to A.B.)

\section{Author Contributions}

A.B. provided the experimental data and figure. A.B. and N.P. wrote and edited the manuscript.

\section{References}

1 Szeto HH, Liu S, Soong Y, Seshan SV, CohenGould L, Manichev V, et al. Mitochondria protection after acute ischemia prevents prolonged upregulation of IL- $1 \beta$ and IL-18 and arrests CKD. J Am Soc Nephrol. 2017;28(5): 1437-49.

2 Perico L, Morigi M, Rota C, Breno M, Mele C, Noris M, et al. Human mesenchymal stromal cells transplanted into mice stimulate renal tubular cells and enhance mitochondrial function. Nat Commun. 2017;8(1):983.

3 Rousselle TV, Kuscu C, Kuscu C, Schlegel K, Huang L, Namwanje M, et al. Fty720 regulates mitochondria biogenesis in dendritic cells to prevent kidney ischemic reperfusion injury. Front Immunol. 2020;11:1278.

4 Doulamis IP, Guariento A, Duignan T, Kido T, Orfany A, Saeed MY, et al. Mitochondrial transplantation by intra-arterial injection for acute kidney injury. Am J Physiol Renal Physiol. 2020;319(3):F403-13.

5 Hall AM, Rhodes GJ, Sandoval RM, Corridon PR, Molitoris BA. In vivo multiphoton imaging of mitochondrial structure and function during acute kidney injury. Kidney Int. 2013; 83(1):72-83.

6 Brooks C, Wei Q, Cho SG, Dong Z. Regulation of mitochondrial dynamics in acute kidney injury in cell culture and rodent models. J Clin Invest. 2009;119(5):1275-85.
7 Bonventre JV, Yang L. Cellular pathophysiology of ischemic acute kidney injury. J Clin Invest. 2011;121(11):4210-21.

8 Zhang Q, Raoof M, Chen Y, Sumi Y, Sursal T, Junger $\mathrm{W}$, et al. Circulating mitochondrial damps cause inflammatory responses to injury. Nature. 2010;464(7285):104-7.

9 Emani SM, Piekarski BL, Harrild D, Del Nido PJ, McCully JD. Autologous mitochondrial transplantation for dysfunction after ischemia-reperfusion injury. J Thorac Cardiovasc Surg. 2017;154(1):286-9.

10 Preble JM, Pacak CA, Kondo H, MacKay AA, Cowan DB, McCully JD. Rapid isolation and purification of mitochondria for transplantation by tissue dissociation and differential filtration. J Vis Exp. 2014(91):e51682.

11 Masuzawa A, Black KM, Pacak CA, Ericsson M, Barnett RJ, Drumm C, et al. Transplantation of autologously derived mitochondria protects the heart from ischemia-reperfusion injury. Am J Physiol Heart Circ Physiol. 2013; 304(7):H966-82.

12 Kaza AK, Wamala I, Friehs I, Kuebler JD, Rathod RH, Berra I, et al. Myocardial rescue with autologous mitochondrial transplantation in a porcine model of ischemia/reperfusion. J Thorac Cardiovasc Surg. 2017;153(4):93443.
13 Jabbari H, Roushandeh AM, Rostami MK, Razavi-Toosi MT, Shokrgozar MA, JahanianNajafabadi A, et al. Mitochondrial transplantation ameliorates ischemia/reperfusion-induced kidney injury in rat. Biochim Biophys Acta Mol Basis Dis. 2020;1866(8):165809.

14 Lin HC, Liu SY, Lai HS, Lai IR. Isolated mitochondria infusion mitigates ischemia-reperfusion injury of the liver in rats. Shock. 2013; 39(3):304-10.

15 Zhu L, Zhang J, Zhou J, Lu Y, Huang S, Xiao $\mathrm{R}$, et al. Mitochondrial transplantation attenuates hypoxic pulmonary hypertension. Oncotarget. 2016;7(31):48925-40.

16 McCully JD, Cowan DB, Pacak CA Toumpoulis IK, Dayalan H, Levitsky S. Injection of isolated mitochondria during early reperfusion for cardioprotection. Am J Physiol Heart Circ Physiol. 2009;296(1):H94-105.

17 Kesner EE, Saada-Reich A, Lorberboum-Galski H. Characteristics of mitochondrial transformation into human cells. Sci Rep. 2016;6: 26057.

18 Sinclair KA, Yerkovich ST, Hopkins PM, Chambers DC. Characterization of intercellular communication and mitochondrial donation by mesenchymal stromal cells derived from the human lung. Stem Cell Res Ther. 2016;7(1):91. 